\title{
Science applications of the Montage image mosaic engine
}

\author{
G. Bruce Berriman ${ }^{1}$, Anastasia C. Laity ${ }^{1}$, John C. Good ${ }^{1}$, \\ Daniel S. Katz ${ }^{2}$, Joseph C. Jacob ${ }^{2}$, Ewa Deelman ${ }^{3}$, Gurmeet Singh ${ }^{3}$, \\ Mei-Hu $\mathrm{Su}^{3}$ and Thomas A. Prince ${ }^{4}$ \\ ${ }^{1}$ Infrared Processing and Analysis Center, Caltech, MS 100-22, Pasadena, CA 91125, USA \\ email: gbb,laity,jeg@ipac.caltech.edu \\ ${ }^{2}$ Jet Propulsion Laboratory, 4800 Oak Grove Drive, Pasadena, CA 91109, USA \\ ${ }^{3}$ USC Information Sciences Institute, 4676 Admiralty Way, Marina del Rey, CA 90292, USA \\ ${ }^{4}$ Division of Physics, Mathematics and Astronomy, Caltech, Pasadena, CA 91125, USA
}

Montage is a portable, scaleable toolkit that runs on end-users desktops, clusters, and computing grids. It generates astronomical image mosaics that preserve the calibration and photometry in the input FITS files. The code is available for download at the project website at ¡http://montage.ipac.caltech.edui, and includes independent modules for image discovery (including support for VO image access protocols), image reprojection, rectification of the sky background to a common level and image co-addition.

Montage is in active use to support product generation, and in generation of products for use in on-line data access services. Customers are taking advantage of the modular design, and are in some cases using Montage as a reprojection engine, or as a background rectification engine. Three Spitzer Space Telescope Legacy teams, the Galactic Legacy Infrared Mid Plane Survey Extraordinaire (GLIMPSE), the Spitzer Wide-Area Infrared Extragalactic Survey (SWIRE) and Surveying the Agents of a Galaxy's Evolution (SAGE), have integrated Montage into their pipelines, where it supports the generation of science products for public release. The INT/WFC Photometric $\mathrm{H} \alpha$ Survey (IPHAS) of the Northern Galactic Plane are making similar use of Montage. The Visible and Infrared Survey Telescope for Astronomy (VISTA) is evaluating Montage to stitch together image 'pawprints' to produce a fully-sampled tile under evaluation. The COSMOS Hubble Treasury Program used Montage to validate Advanced Camera for Surveys (HST-ACS) image mosaics generated at the Hubble Space Telescope Science Institute. The Multi-Mission Archive at Space Telescope (MAST) and the Stellar Archive and Retrieval System (StARS) (unreleased) have used Montage to generate 2MASS postage stamp images centered on their target sources. The NASA/IPAC Infra Red Science Archive is using Montage to create 3-color browse products and support image cutout services. The Spitzer Space Telescope Outreach program is generating mosaics in uncommon projections, often the best ones for E/PO products. Montage components have been used by the NVO Quick Sky Statistics and Sky Coverage Service to Montage components used to determine sky coverage, and to generate single-survey and composite-survey coverage maps.

An on-request image mosaic service is under evaluation by astronomers. This service has already been used by Beaton06 to confirm the presence of a bar in M31. The rectification of the background revealed the 'boxy' signature characteristic of a bar.

\section{Reference}

Bearman, R., et al. 2006, ApJ (Letters), in press 